OPEN ACCESS

Edited by:

Fei Gao,

Beijing Genomics Institute (BGI), China

Reviewed by:

Palash C. Maity,

Universität UIm, Germany

Gerardo Ferrer,

Josep Carreras Leukaemia Research Institute (IJC), Spain

${ }^{*}$ Correspondence: $\mathrm{Jin} \mathrm{He}$

hejin1@msu.edu

Specialty section: This article was submitted to Hematologic Malignancies, a section of the journal

Frontiers in Oncology

Received: 05 August 2021 Accepted: 17 September 2021

Published: 08 October 2021

Citation:

Aljazi MB, Gao Y, Wu Y, Mias Gl and He J (2021) Histone H3K36me2-

Specific Methyltransferase ASH1L Promotes MLLAF9-Induced Leukemogenesis.

Front. Oncol. 11:754093. doi: 10.3389/fonc.2021.754093

\section{Histone H3K36me2-Specific Methyltransferase ASH1L Promotes MLL-AF9-Induced Leukemogenesis}

\author{
Mohammad B. Aljazi ${ }^{1}$, Yuen Gao ${ }^{1}$, Yan $W^{1}{ }^{1}$, George I. Mias ${ }^{1,2}$ and Jin $\mathrm{He}^{1 *}$ \\ ${ }^{1}$ Department of Biochemistry and Molecular Biology, College of Nature Sciences, Michigan State University, East Lansing, \\ MI, United States, 2 Institute for Quantitative Health Science and Engineering, Michigan State University, East Lansing, MI, \\ United States
}

ASH1L and MLL1 are two histone methyltransferases that facilitate transcriptional activation during normal development. However, the roles of $\mathrm{ASH} 1 \mathrm{~L}$ and its enzymatic activity in the development of MLL-rearranged leukemias are not fully elucidated in Ash1L gene knockout animal models. In this study, we used an Ash1L conditional knockout mouse model to show that loss of ASH1L in hematopoietic progenitor cells impaired the initiation of MLL-AF9-induced leukemic transformation in vitro. Furthermore, genetic deletion of ASH1L in the MLL-AF9-transformed cells impaired the maintenance of leukemic cells in vitro and largely blocked the leukemia progression in vivo. Importantly, the loss of $\mathrm{ASH} 1 \mathrm{~L}$ function in the Ash1L-deleted cells could be rescued by wild-type but not the catalytic-dead mutant $\mathrm{ASH} 1 \mathrm{~L}$, suggesting the enzymatic activity of $\mathrm{ASH} 1 \mathrm{~L}$ was required for its function in promoting MLL-AF9-induced leukemic transformation. At the molecular level, ASH1L enhanced the MLL-AF9 target gene expression by directly binding to the gene promoters and modifying the local histone H3K36me2 levels. Thus, our study revealed the critical functions of $\mathrm{ASH} 1 \mathrm{~L}$ in promoting the MLL-AF9-induced leukemogenesis, which provides a molecular basis for targeting $\mathrm{ASH} 1 \mathrm{~L}$ and its enzymatic activity to treat MLL-AF9-induced leukemias.

Keywords: MLL1, ASH1L, histone modification, H3K36me2, leukemogenesis, MLL-AF9 fusion

\section{INTRODUCTION}

The MLL rearrangement (MLLr) caused by 11q23 chromosomal translocations creates a variety MLL fusion proteins that drive the acute lymphoblastic and myeloid leukemia development, which accounts for approximate 5-10\% acute leukemias in human patients (1-5). Despite recent progression in the development of chemotherapies against leukemias, the overall prognosis for the MLLr leukemias remains poor $(6,7)$.

MLL1 protein is a histone lysine methyltransferase (KMTase) that contains a SET (Su(var)3-9, Enhancer-of-zeste and Trithorax) domain to catalyze trimethylation of histone H3 lysine 4 (H3K4me3) (8). Functionally, MLL1 belongs to the Trithorax-group (TrxG) proteins that antagonize the Polycomb-group $(\mathrm{PcG})$-mediated gene silencing and facilitate transcriptional activation (9). In 11q23 chromosomal translocations, the $N$-terminal portion of MLL1 is fused 
with a variety of fusion partners to generate different oncogenic MLL fusion proteins that function as disease drivers leading to leukemia development (10-12). Previous studies have revealed that the $N$-terminal portion of MLL fusion proteins interacts with MENIN and LEDGF (Lens Epithelium-Derived Growth Factor), which is critical for the recruitment of MLL fusion proteins to chromatin, whereas the $C$-terminal fusion partners interact with various trans-activators to induce transcriptional activation (13-17). However, since the MLL fusion proteins lack the intrinsic histone $\mathrm{H} 3 \mathrm{~K} 4$ methyltransferase activity due to loss of the SET domain located in the C-terminal portion of MLL1 (10), it is unclear whether other histone modifications are required for the MLL fusion proteins-induced gene expression and leukemogenesis.

Recently, another member of TrxG proteins, ASH1L (Absent, Small, or Homeotic-Like 1), was found to play important roles in normal hematopoiesis and leukemogenesis $(8,18,19)$. Biochemically, ASH1L is a histone KMTase that mediates dimethylation of histone H3 lysine 36 (H3K36me2) (20). Similar to MLL1, ASH1L facilitates gene expression through antagonizing PcG-mediated gene silencing (8). Previous studies have shown that ASH1L and MLL1 co-occupies the same transcriptional regulatory regions, and loss of either ASH1L or MLL1 reduces the expression of common genes (21-23), suggesting ASH1L and MLL1 function synergistically to activate gene expression during normal development. However, the significance of ASH1L and its-mediated histone H3K36me2 in the MLLr-associated leukemogenesis has not been addressed in the Ash1L gene knockout animal models.

In this study, we used an $A \operatorname{sh} 1 L$ conditional knockout mouse model to show that loss of ASH1L in hematopoietic progenitor cells (HPCs) impaired the initiation of MLL-AF9-induced leukemic transformation in vitro. Furthermore, genetic deletion of ASH1L in the MLL-AF9-transformed cells impaired the maintenance of leukemic cells in vitro and largely blocked the leukemia progression in vivo. Importantly, the loss of ASH1L function in the Ash1L-deleted cells could be rescued by ectopic expression of wild-type but not the catalytic-dead mutant ASH1L, suggesting the enzymatic activity of ASH1L was required for its function in promoting MLL-AF9-induced leukemic transformation. At the molecular level, ASH1L activated the MLL-AF9 target gene expression by directly binding to the gene promoters and modifying the local histone H3K36me2 levels. Thus, our study revealed the critical functions of ASH1L in MLL-AF9-induced leukemogenesis and raised the possibility that ASH1L might serve as a potential therapeutic target for the treatment of MLL-AF9-induced leukemias.

\section{MATERIALS AND METHODS}

\section{Mice}

The Ash1L conditional knockout mice were generated as previously reported (24). To generate inducible Ash1L deletion, mice were crossed with Rosa26-CreER ${ }^{\mathrm{T} 2}$ mice that were obtained from The Jackson Laboratory. All mice for this study were backcrossed to $\mathrm{C} 57 \mathrm{BL} / 6$ mice for at least five generations to reach pure genetic background prior to conducting experiments. All mouse experiments were performed with the approval of the Michigan State University Institutional Animal Care \& Use Committee.

\section{Hematopoietic Progenitor Isolation and Culture}

Hematopoietic progenitor cells were isolated from femurs of 4to 6 -week C57BL/6 mice. The red blood cells in the bone marrows were lysed by ammonium chloride solution (Stem Cell Technologies 07800) and filtered with a 70- $\mu \mathrm{m}$ nylon filter. The c-KIT ${ }^{+}$HPCs were isolated using c-KIT antibodyconjugated IMag (BD Biosciences) beads. HPCs cells were maintained in RMPI1640 medium supplemented with 10\% FBS, 1\% MEM non-essential amino acids, 1\% Glutamax, 10 $\mathrm{ng} / \mathrm{mL}, 2$-mercaptoethanol, and $50 \mathrm{ng} / \mathrm{mL} \mathrm{mSCF}$ (PeproTech), $10 \mathrm{ng} / \mathrm{mL} \mathrm{mIL}-6$ (PeproTech), and $10 \mathrm{ng} / \mathrm{mL} \mathrm{mIL}-3$ (PeproTech). To induce CRE-mediated recombination in vitro, 4-hydroxy-tamoxifen (Sigma-Aldrich) was resuspended in DMSO and supplemented into the culture medium with concentration of $250 \mathrm{nM}$.

\section{Retroviral and Lentiviral Vector Production and Transduction}

The pMIG-FLAG-MLL-AF9 retroviral vectors as obtained from Addgene (Plasmid \#71443). Retroviral vectors were generated by co-transfection of retroviral vectors with pGag-pol, pVSVG 293T cells using CalPhos mammalian transfection kit (TaKaRa). After $48 \mathrm{hrs}$ post transfection, viral supernatant was harvested, filtered through a $0.45 \mu \mathrm{m}$ membrane, and concentrated by ultracentrifugation. The lentiviral system was obtained from the National Institutes of Health AIDS Research and Reference Reagent Program. To generate GFP expression vectors, the GFP cDNA was PCR amplified, fused with P2A and puromycin resistant cassette and cloned into the SpeI/EcoRI sites under the EF1 $\alpha$ promoter. To generate lentiviral viruses, the transducing vectors pTY, pHP and pHEF1 $\alpha-$ VSVG were cotransfected into HEK293T cells. The supernatant was collected at 24, 36 and 48 hours after transfection, filtered through a $0.45 \mu \mathrm{m}$ membrane and concentrated by ultracentrifugation. Retroviral and lentiviral transduction of HPCs was performed by spin inoculation for 1 hour at 800g, in RMPI1640 medium supplemented with 10\% FBS, 1x MEM non-essential amino acids (Life Technologies), 1x Glutamax (Life Technologies), 1x sodium pyruvate (Life Technologies), and $10 \mathrm{ng} / \mathrm{mL} \mathrm{mIL}$ 3 (PeproTech).

\section{Serial Methylcellulose Replating Assay and Leukemia Transplantation}

The colony formation assays were conducted by plating 500 cells into methylcellulose media consisting of Iscove MDM (Life Technologies) supplemented with FBS, BSA, insulin-transferrin (Life Technologies), 2-mercaptoethanol, $50 \mathrm{ng} / \mathrm{mL} \mathrm{mSCF}$ (PeproTech), 10 ng/mL mIL-6 (PeproTech), 10 ng/mL mIL-3 (PeproTech), and $10 \mathrm{ng} / \mathrm{mL}$ GM-CSF (PeproTech). After 7-10 
days, the colony numbers were counted under a microscope. The colonies were picked up, and cells were pooled and replated onto secondary methylcellulose plates. Three rounds of replating were performed for each experiment. For leukemia transplantation, recipient $\mathrm{C} 57 \mathrm{BL} / 6$ mice were subjected to total body irradiation at a dose of $11 \mathrm{~Gy}$ with the use of a X-RAD 320 biological irradiator. Donor cells $\left(5 \times 10^{5}\right)$ and radiation protector cells $(5 \times$ $10^{5}$ ) isolated from BM were mixed in $1 \times$ PBS and transplanted into the recipient mice through retro-orbital injection. The mice were fed with water supplemented with trimethoprim/ sulfamethoxazole for 4 weeks after transplantation.

\section{FACS Analysis}

For FACS analysis, cells were stained with antibodies in staining buffer ( $1 \times$ PBS, $2 \% \mathrm{FBS})$ and incubated at $4^{\circ} \mathrm{C}$ for 30 minutes. The samples were washed once with staining buffer before subjected to FACS analysis with the use of a BD LSRII. The antibodies used in this study include anti-Mac-1(eBioscience), anti-Gr-1(eBioscience), anti-c-KIT (eBioscience).

\section{Western Blot Analysis}

Total proteins were extracted by RIPA buffer and separated by electrophoresis by $8-10 \%$ PAGE gel. The protein was transferred to the nitrocellulose membrane and blotted with primary antibodies. The antibodies used for Western Blot and IPWestern Blot analyses included: rabbit anti-ASH1L (1:1000, in house) (24) and IRDye 680 donkey anti-rabbit second antibody (1: 10000, Li-Cor). The images were developed by Odyssey LiCor Imager (Li-Cor).

\section{Quantitative RT-PCR and ChIP-qPCR Assays}

RNA was extracted and purified from cells with the use of Qiashredder (QIAGEN) and RNeasy (QIAGEN) spin columns. Total RNA $(1 \mu \mathrm{g})$ was subjected to reverse transcription using Iscript reverse transcription supermix (Bio-Rad). cDNA levels were assayed by real-time PCR using iTaq universal SYBR green supermix (Bio-Rad) and detected by CFX386 Touch Real-Time PCR detection system (Bio-Rad). Primer sequences for qPCR are listed in Supplementary Table 3. The expression of individual genes is normalized to expression level of Gapdh. ChIP assays that used rabbit anti-ASH1L antibody (in house), rabbit antiH3K36me2 antibody (Abcam), rabbit anti-Flag antibody (Cell Signaling) were carried out according to the previously reported protocol with the following modifications (25): 2 ug antibodies were used in the immunoprecipitation, and chromatin-bound beads were washed 3 times each with TSEI, TSEII, and TESIII followed by 2 washes in $10 \mathrm{mM}$ Tris, $\mathrm{pH} 7.5,1 \mathrm{mM}$ EDTA. Histone modification ChIPs were carried out as previously reported (26). DNA that underwent ChIP was analyzed by quantitative PCR (qPCR), and data are presented as the percentage of input as determined with CFX manager 3.1 software. The amplicons were designed to locate at $1.0-\mathrm{kb}$ upstream of transcriptional starting sites (TSS) and transcription ending sties (TES) of Hoxa9/Hoxa10 genes. The mouse intracisternal A-particle LTR repeat elements were included as a negative control for the ASH1L binding. The ChIP primers for the mouse IAP LTR were purchased from Cell Signaling (85916, Cell Signaling). Other qPCR and ChIP primers are listed in Supplementary Table 3, respectively.

\section{RNA-Seq Sample Preparation for HiSeq4000 Sequencing}

RNA was extracted and purified from cells using QI shredder (Qiagen) and RNeasy (Qiagen) spin columns. Total RNA (1 $\mu \mathrm{g})$ was used to generate RNA-seq library using NEBNext Ultra Directional RNA library Prep Kit for Illumina (New England BioLabs, Inc) according to the manufacturer's instructions. Adapter-ligated cDNA was amplified by PCR and followed by size selection using agarose gel electrophoresis. The DNA was purified using Qiaquick gel extraction kit (Qiagen) and quantified both with an Agilent Bioanalyzer and Invitrogen Qubit. The libraries were diluted to a working concentration of $10 \mathrm{nM}$ prior to sequencing. Sequencing on an Illumina HiSeq4000 instrument was carried out by the Genomics Core Facility at Michigan State University.

\section{RNA-Seq Data Analysis}

RNA-Seq data analysis was performed essentially as described previously. All sequencing reads were mapped $\mathrm{mm} 9$ of the mouse genome using Tophat2 (27). The mapped reads were normalized to reads as Reads Per Kilobase of transcript per Million mapped reads (RPKM). The differential gene expression was calculated by Cuffdiff program and the statistic cutoff for identification of differential gene expression is $\mathrm{p}<0.01$ and 1.5fold RPKM change between samples (28). The heatmap and plot of gene expression were generated using plotHeatmap and plotProfile in the deepTools program (29). The differential expressed gene lists were input into the David Functional Annotation Bioinformatics Microarray Analysis for the GO enrichment analyses (https://david.ncifcrf.gov/).

\section{Statistical Analysis}

All statistical analyses were performed using GraphPad Prism 9 (GraphPad Software). Parametric data were analyzed by a twotailed $t$ test or two-way ANOVA test for comparisons of multiple samples. The post-transplantation survivals were analyzed by the Gehan-Breslow-Wilcoxon test. $P$ values $<0.05$ were considered statistically significant. Data are presented as mean \pm SEM.

\section{RESULTS}

\section{ASH1L Promotes the Initiation of MLL- AF9-Induced Leukemic Transformation In Vitro}

To examine the function of ASH1L in MLL-AF9-induced leukemogenesis, we generated an $A s h 1 L$ conditional knockout (Ash1L-cKO) mouse line in which two LoxP elements inserted into the exon 4 flanking regions (24). A CRE recombinasemediated deletion of exon 4 resulted in altered splicing of mRNA that created a premature stop codon before the sequences encoding the first functional AWS (associated with SET) 
domain (Figures 1A, B). The Ash $1 L$-cKO mice were further crossed with the Rosa26-CreER ${ }^{\mathrm{T} 2}$ mice to generate a tamoxifeninducible $A s h 1 L$ knockout line $\left(A \operatorname{sh} 1 L^{2 \mathrm{f} / 2 \mathrm{f}}\right.$;Rosa26-CreER $\left.{ }^{\mathrm{T} 2}\right)$, which allowed us to study the function of ASH1L in leukemogenesis in vitro and in vivo.
Using this $A s h 1 L$-cKO mouse model, we investigate the role of ASH1L in the initiation of MLL-AF9-induced leukemic transformation. To this end, we isolated the bone marrow cells from wild-type $\left(A \operatorname{sh} 1 L^{+/+} ; C r e-E R^{T 2}\right)$ and Ash $1 L$-cKO $\left(A \operatorname{sh} 1 L^{2 \mathrm{f} / 2 \mathrm{f}}\right.$; Cre- $E R^{T 2}$ ) mice, respectively. The $\mathrm{c}-\mathrm{KIT}^{+}$HPCs were further
A

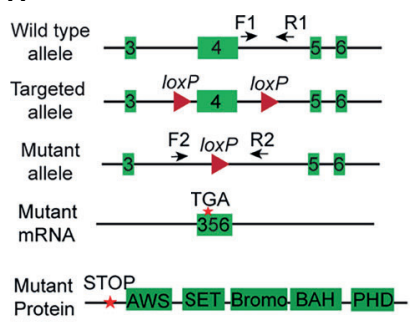

B

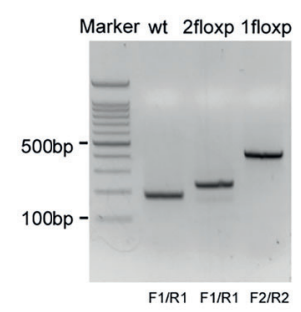

C

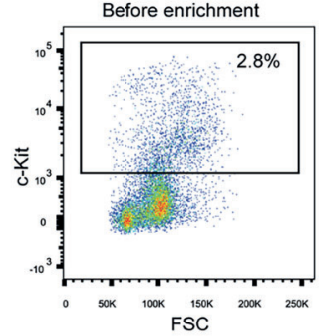

D
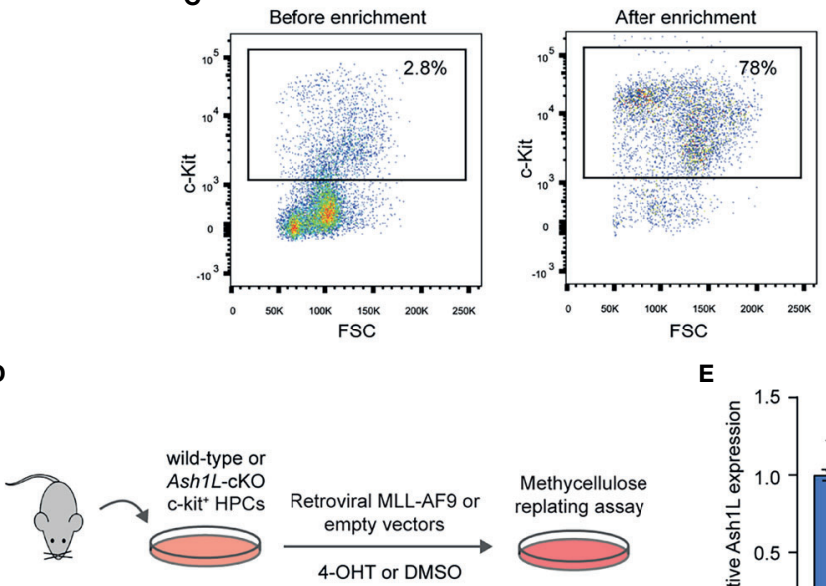

Methycellulose replating assay

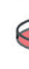

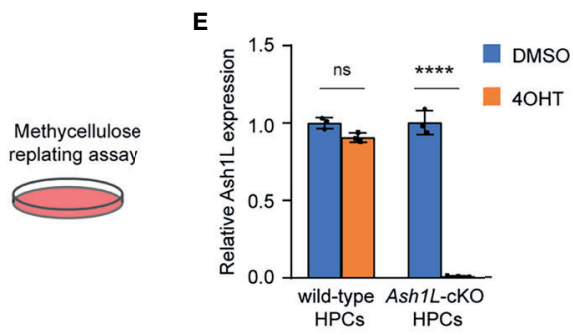

$\mathbf{F}$

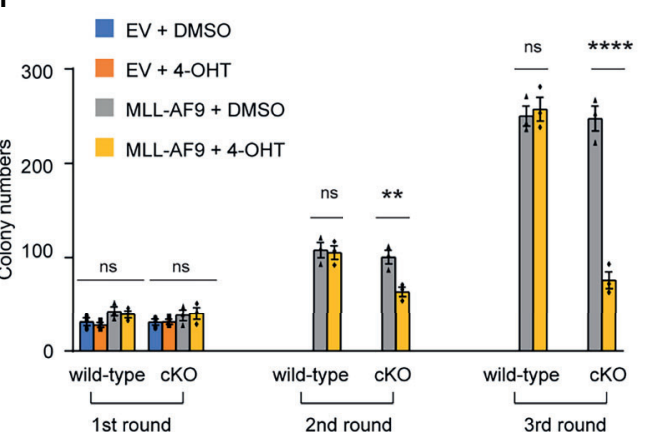

G
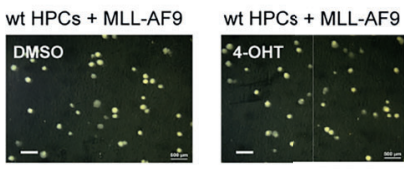

CKO HPCs+ MLL-AF9

cKO HPCs+ MLL-AF9
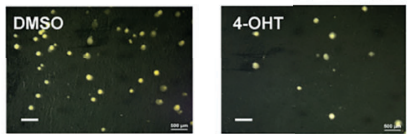

FIGURE 1 | ASH1L is required for the initiation of MLL-AF9-induced leukemic transformation. (A) Diagram showing the strategy for the generation of Ash1L conditional knockout mice. CRE-mediated deletion of exon 4 results in an altered spliced mRNA with a premature stop codon, which generates a truncated protein without all functional AWS, SET, Bromo, BAH and PHD domains. The arrows labeled as F and R represent the genotyping primers. (B) Genotyping results showing the PCR results of wild-type, 2 floxP, and 1 floxP alleles. (C) FACS analysis showing the C-KIT+ HPC populations before and after enrichment with C-KIT antibodyconjugated beads. (D) Schematic experimental procedure. (E) qRT-PCR analysis showing the Ash1L expression levels in wild-type and Ash1L-cKO cells after treated with 4-OHT or DMSO. The results were normalized against levels of Gapdh and the expression level in DMSO-treated cells was arbitrarily set to 1. The error bars represent mean \pm SEM, $n=3$ per group. ${ }^{\star \star \star \star} P<0.0001$, ns, not significant. (F) Methylcellulose replating assays showing the colony numbers for each round of plating. The error bars represent mean \pm SEM, $n=3$ per group. ${ }^{\star \star} P<0.01$; ${ }^{\star \star \star \star} P<0.0001$, ns, not significant. (G) Photos showing the representative colony formation on methylcellulose plates for each group. $\mathrm{Bar}=0.5 \mathrm{~mm}$. 
enriched by the c-KIT antibody-conjugated magnetic beads (Figure 1C). The HPCs were cultured in the HPC medium supplemented with murine IL-3, IL-6, and SCF for three days, and followed by transduction of retroviral vectors expressing a MLL1-AF9 fusion gene or control empty viruses (EV). After transduction, the cells were cultured in the suspension medium with 4-hydroxytamoxifen (4-OHT) for five days to induce Ash1L gene deletion in the Ash $1 L$-cKO HPCs (Figure 1D). The quantitative RT-PCR (qRT-PCR) analysis showed that the Ash1L expression reduced to less than $5 \%$ at the mRNA level in the Ash $1 L$ deleted cells (Figure 1E). To investigate the effect of Ash1L loss on the initiation of MLL-AF9-induced leukemic transformation in vitro, we performed serial colony replating assays by plating the cells on the semi-solid methylcellulose medium to examine the leukemic transformation. The results showed that although the cells transduced with $M L L-A F 9$ or empty vectors had comparable colony numbers in the first round of plating, the cells transduced with control empty vectors did not form colonies in the following rounds of replating. In contrast, both wild-type and Ash $1 L$-cKO HPCs transduced with $M L L-A F 9$ retroviruses formed colonies in all three rounds of plating, indicating successful leukemic transformation by the MLL-AF9 transgene in vitro. Notably, compared to the MLLAF9-transduced wild-type cells, the Ash1L-deleted cells had reduced colony numbers in the second and third rounds of plating, suggesting that loss of Ash1L in HPCs compromised the MLLAF9-induced leukemic transformation (Figures 1F, G), suggesting ASH1L promotes the MLL-AF9-induced leukemic transformation in vitro.

\section{ASH1L Facilitates the Maintenance of MLL-AF9-Induced Leukemic Cells In Vitro}

Next, we examined the functional role of $A s h 1 L$ in maintaining the MLL-AF9-transformed cells. To this end, we transduced both wild-type and $A$ sh $1 L$-cKO HPCs with $M L L-A F 9$ retroviruses and plated the transduced cells onto the methylcellulose medium. After three rounds of replating, the transformed colonies were manually picked and cultured in the suspension medium supplemented with 4-OHT for 5 days to induce deletion of Ash $1 L$ in the Ash $1 L$-cKO cells. The cells were further maintained in suspension culture without 4-OHT for 5 days before plated onto the methylcellulose to examine the colony formation (Figure 2A). The results showed that compared to the wildtype MLL-AF9-transformed cells, the Ash1L-deleted cells had marked reduced colony formation (Figures 2B, C), suggesting that ASH1L facilitated the maintenance of MLL-AF9 transformed cells in vitro.

To examine cellular responses to the Ash $1 L$ depletion, we performed the FACS analysis to examine cell death in response

A

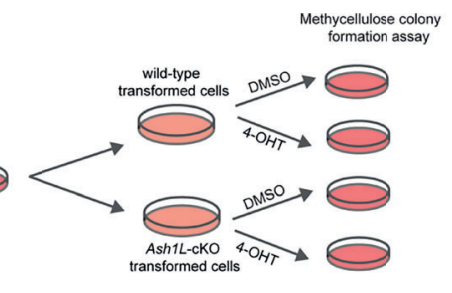

B

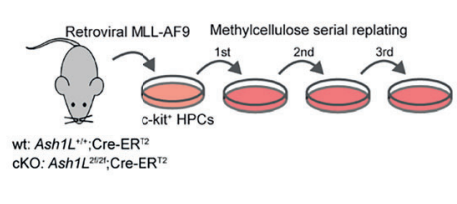

C
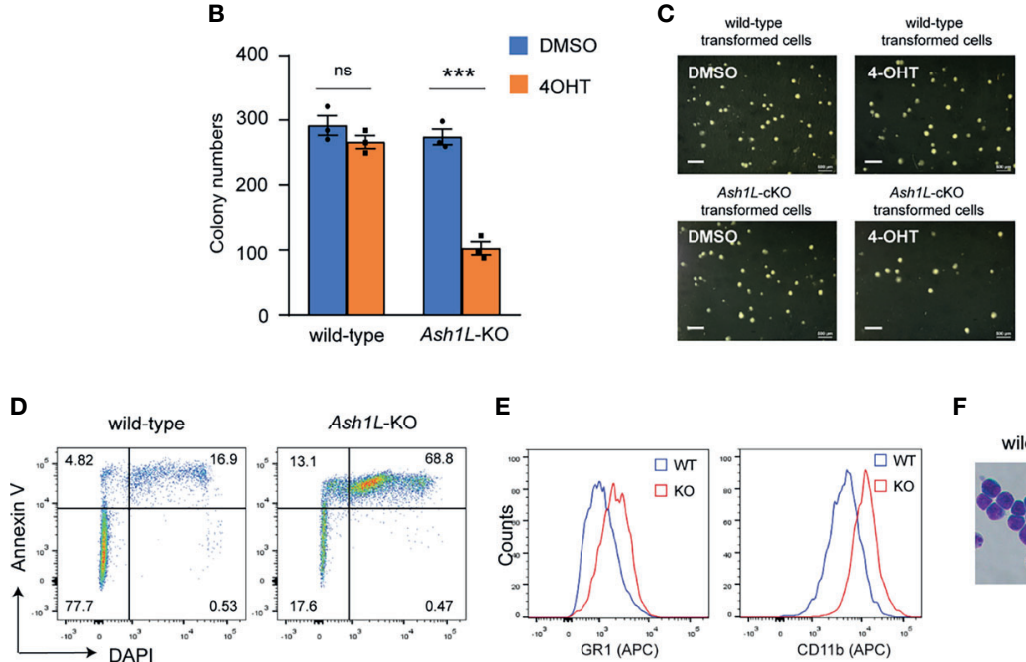

E

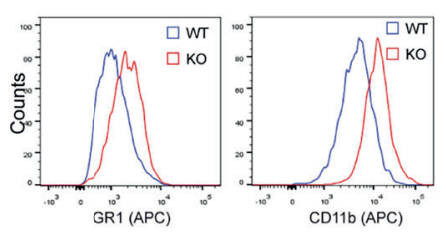

$\mathbf{F}$

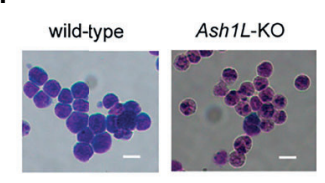

FIGURE 2 | ASH1L is required for the maintenance of MLL-AF9-induced leukemic cells in vitro. (A) Schematic experimental procedure. (B) Methylcellulose colony formation assays showing the colony numbers. The error bars represent mean $\pm \mathrm{SEM}, \mathrm{n}=3$ per group. ${ }^{\star \star *} P<0.001$; ns, not significant. (C) Photos showing the representative colony formation on methylcellulose plates for each group. Bar $=0.5 \mathrm{~mm}$. (D) Representative FACS results showing the Annexin $\mathrm{V}+$ and DAPI+ populations of wild-type and Ash1L-KO MLL-AF9-transformed cells. (E) Representative FACS results showing the GR-1 and CD11b expression of wild-type and Ash1L-KO MLL-AF9-transformed cells. (F) Photos showing the Wright-Giemsa staining of wild-type and Ash1L-KO MLL-AF9-transformed cells. Bar $=10 \mu \mathrm{m}$. 
to the loss of Ash1L in the MLL-AF9-transformed cells. The results showed that compared to the wild-type cells, the Ash $1 L$-deleted cells had increased populations of both early apoptotic cells (Annexin V+/DAPI-) and late dead cells (Annexin V+/DAPI+) (Figure 2D), suggesting that the loss of $A s h 1 L$ induced cell death of MLL-AF9-transformed cells. Moreover, FACS analyses showed that compared to the wild-type transformed cells, the Ash $1 L$ deleted cells had increased expression of myeloid differentiation surface markers CD11b and GR-1 (Figure 2E). Morphologically, the wild-type transformed cells displayed leukoblast-like morphology with enlarged dark stained nuclei, while the Ash1Ldeleted cells had light-stained and segmented nuclei, a feature indicating the differentiation towards matured myeloid cells (Figure 2F). Taken together, these results suggested that ASH1L facilitated the maintenance of MLL-AF9-transformed cells through suppressing cell death and differentiation.

\section{ASH1L Promotes the MLL-AF9-Induced Leukemia Development In Vivo}

To determine the role of ASH1L in the MLL-AF9-induced leukemogenesis in vivo, we performed leukemia transplantation assays and monitor the leukemia development in recipient mice. To this end, the wild-type and Ash1L-deleted MLL-AF9transformed cells were labeled with GFP by transduction with lentiviral-GFP vectors, mixed with normal protective bone marrow cells, and transplanted into the total-body-irradiated (TBI) syngeneic recipient mice (Figure 3A). Four weeks after transplantation, FACS analysis showed that the mice transplanted with wild-type leukemic cells had higher GFP+ leukemic cell populations in the peripheral blood compared to the mice received with $A$ sh $1 L$-KO leukemic cells (Figure 3B), which was consistent with the higher leukemic cell numbers in the peripheral blood smears and splenomegaly found in the mice transplanted with wild-type leukemic cells (Figures 3C, D). All mice transplanted with wild-type leukemic cells died within 3 months after transplantation, and the median survival time was around 8.5 weeks. In contrast, the mice transplanted with Ash1L-deleted cells had significant longer survival time (Chi square $=10.73, \mathrm{df}=1, p=$ 0.0011) compared to the mice transplanted with wild-type leukemic cells (Figure 3E). These results suggested that ASH1L in the MLL-AF9-transformed leukemic cells promoted the development and progression of leukemia in vivo.

\section{The Enzymatic Activity of ASH1L Is Required for Its Function in Promoting MLL-AF9-Induced Leukemic Transformation}

Next, we set out to determine whether the histone methyltransferase activity of ASH1L was required for its function in promoting MLLAF9-induced leukemic transformation. To this end, the Ash $1 L$-cKO HPCs were infected with retroviruses expressing $M L L-A F 9$ transgene, followed by transduced with lentiviral vectors expressing either wild-type ASH1L or catalytic-dead mutant ASH1L(H2214A) (21). The transformed cells were treated with 4$\mathrm{OHT}$ to induce deletion of endogenous Ash1L gene (Figure 4A). Western blot analysis showed that both wild-type and mutant exogenous ASH1L had a similar expression level (Figure 4B). The cells were further plated onto the methylcellulose medium to examine the colony formation (Figure 4A). The results showed that compared to the wild-type ASH1L-expressed cells, the cells with
A

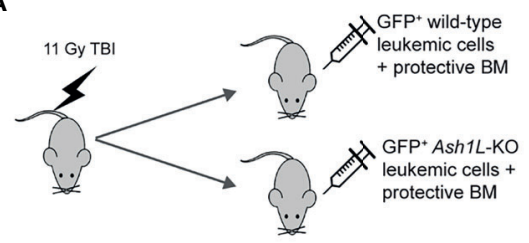

C

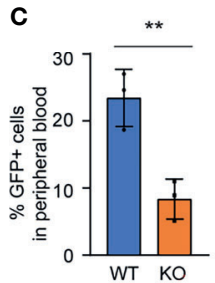

B

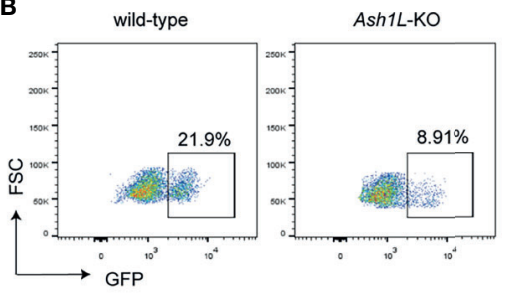

E

$\mathbf{F}$

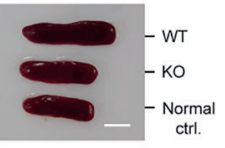

FIGURE 3 | ASH1L promotes the MLL-AF9-induced leukemia development in vivo. (A) Schematic experimental procedure. (B) Representative FACS analysis showing the GFP+ leukemic cell populations in the peripheral blood of mice transplanted with wild-type or Ash1L-KO MLL-AF9-transformed cells. (C) Quantitative results showing the percentage of GFP+ leukemic cell populations in the peripheral blood of mice transplanted with wild-type or Ash1L-KO MLL-AF9-transformed cells. The error bars represent mean \pm SEM, $\mathrm{n}=3$ per group. ${ }^{\star *} \mathrm{P}<0.01$. (D) Photos showing the leukemic cells in the peripheral blood smear of mice transplanted with wild-type or Ash1L-KO MLL-AF9-transformed cells. Bar $=10 \mu \mathrm{m}$. (E) Photos showing the representative spleen size from the normal control mice (Normal ctrl.), mice transplanted with wild-type (WT) or Ash1L-KO (KO) MLL-AF9-transformed cells. The samples were collected at post-transplantation 4 weeks. Bar $=5$ mm. (F) Kaplan-Meier survival curve of mice transplanted with wild-type or Ash1L-KO MLL-AF9-transformed cells. $P$ value calculated using a Gehan-Breslow-Wilcoxon test. $\mathrm{n}=10$ mice per group. 
A

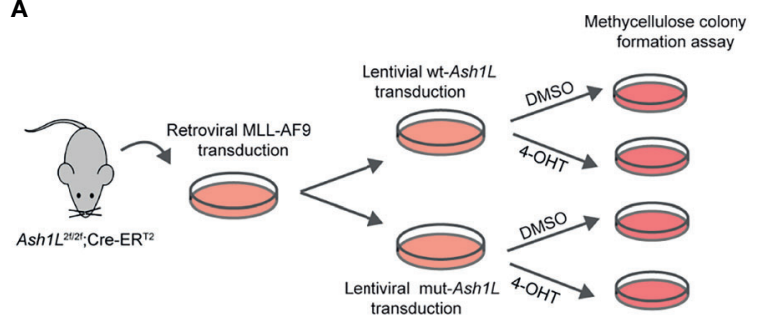

C

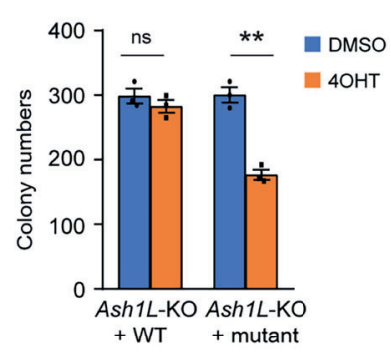

E

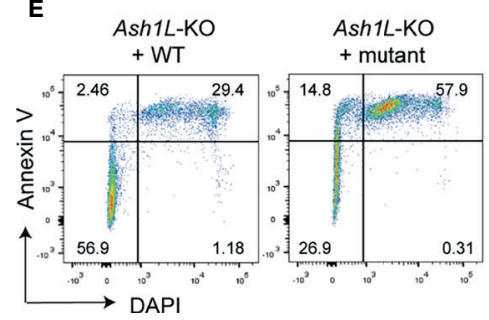

D

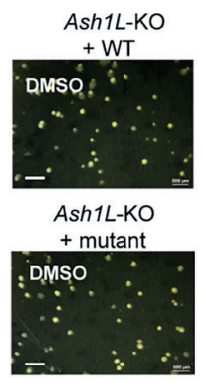

$\mathbf{F}$

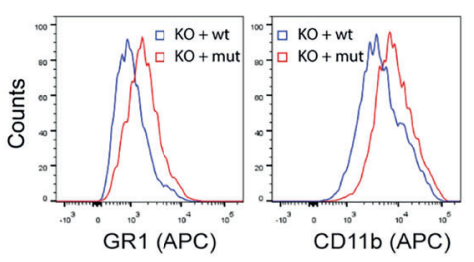

B

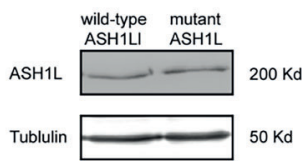

FIGURE 4 | The enzymatic activity of ASH1L is required for its function in promoting MLL-AF9-induced leukemic transformation. (A) Schematic experimental procedure. (B) WB analysis showing the ectopic expression of wild-type and mutant ASH1L. (C) Methylcellulose colony formation assays showing the colony numbers. The error bars represent mean \pm SEM, $n=3$ per group. ${ }^{\star \star} P<0.01$; ns, not significant. (D) Photos showing the representative colony formation on methylcellulose plates for each group. Bar $=0.5 \mathrm{~mm}$. (E) Representative FACS results showing the Annexin V+ and DAPI+ populations of Ash1L-KO cells rescued with wild-type and mutant ASH1L. (F) Representative FACS results showing the GR1 and CD11b expression of Ash1L-KO cells rescued with wild-type and mutant ASH1L.

ectopic expression of catalytic-dead mutant ASH1L had reduced colony formation (Figures 4C, D). Similar to the Ash1L-deleted cells, the Ash1L-deleted cells rescued with mutant ASH1L had increased cell death and upregulated expression of myeloid differentiation markers of CD11b and GR-1 (Figures 4E, F). These results suggested that ASH1L histone methyltransferase activity was required for its function in promoting MLL-AF9induced leukemogenesis by inhibiting cell death and blocking myeloid differentiation.

\section{ASH1L Facilitates the MLL-AF9-Induced Leukemogenic Gene Expression}

To examine the molecular mechanisms underlying the function of ASH1L in promoting MLL-AF9-induced leukemogenesis, we performed RNA-seq analyses to examine the transcriptome changes in normal HPCs, wild-type and Ash1L-deleted MLLAF9-tranformed cells. The results showed that compared to normal HPCs, the MLL-AF9-transformed cells had 1,021 upregulated and 1,228 downregulated genes (cutoff: fold changes $>1.5$, FDR $<0.05$ ), respectively (Figure $\mathbf{5 A}$ ). The gene ontology (GO) enrichment analysis showed that both upregulated and downregulated genes were involved in immune processes and inflammatory responses (cutoff: FDR < 0.05) (Supplementary Tables $\mathbf{1}$ and 2), suggesting that MLLAF9 fusion proteins disrupted the normal differentiation and mis-regulated the normal function of myeloid cells. Notably, multiple genes, such as Hoxa5, Hoxa7, Hoxa9, Hoxa10 and MeisI that were known to mediate the MLL-AF9-induced leukemogenesis, were highly expressed in the MLL-AF9transformed cells (Figure 5B). Further RNA-seq analysis showed that compared to MLL-AF9-transformed wild-type cells, the Ash1L-deleted cells had 372 upregulated gene and 472 downregulated genes (cutoff: fold changes $>1.5$, FDR $<$ 0.05), respectively (Figure 5C). Cross-examining these two data sets revealed that 105 genes, including Hoxa5, Hoxa7, Hoxa9, Hoxa10, and MeisI that were highly expressed in the wild-type MLL-AF9-transformed cells, were downregulated in the Ash1Ldeleted cells (Figures 5D, E). Altogether, these results suggested 


\section{A}

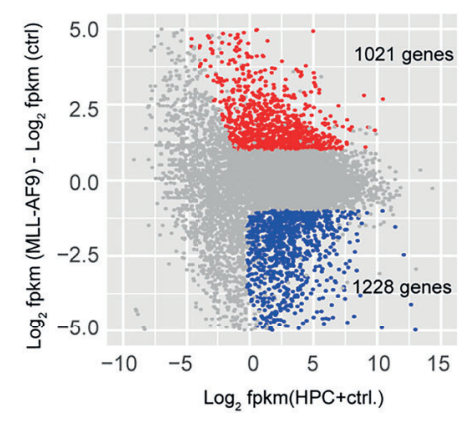

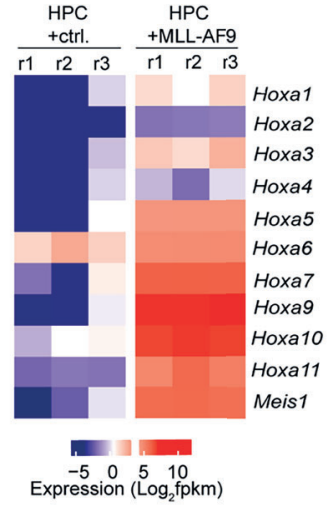

C

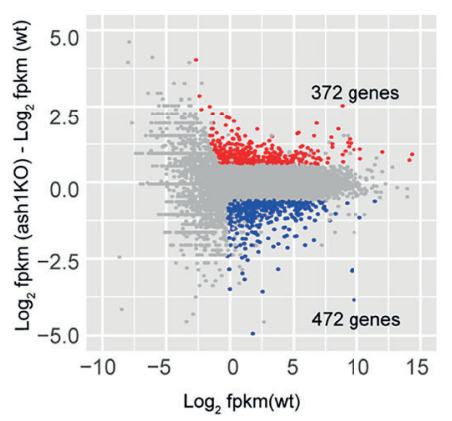

D

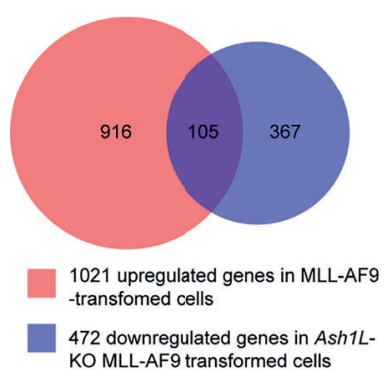

E

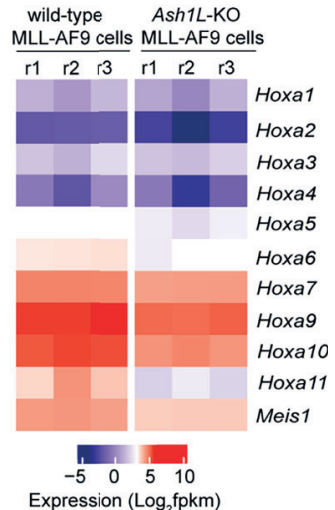

FIGURE 5 | ASH1L facilitates the MLL-AF9-induced leukemogenic gene expression. (A) Plot showing 1021 up- and 1228 down-regulated genes in the MLL-AF9transformed cells compared to the normal HPCs. (B) Heatmap showing the upregulation of Hoxa gene cluster and Meis/ in the MLL-AF9-transformed cells compared to normal HPCs. (C) Plot showing 372 up- and 472 down-regulated genes in the Ash1L-KO MLL-AF9-transformed cells compared to the wild-type MLLAF9-transformed cells. (D) Venn diagram showing the 105 genes upregulated in the MLL-AF9-transformed cells and downregulated in the Ash1L-KO cells.

(E) Heatmap showing the Hoxa gene cluster and Meis/ downregulated in the Ash1L-KO cells compared to the wild-type MLL-AF9-transformed cells.

that ASH1L promoted the MLL-AF9-induced leukemogenesis by facilitating the MLL-AF9-induced leukemic gene expression.

\section{ASH1L Binds and Mediates the Histone H3K36me2 Modification at Hoxa9 and Hoxa10 Gene Promoters}

To determine whether ASH1L directly regulated the expression of MLL-AF9 target genes, we performed chromatin immunoprecipitation (ChIP) coupled with quantitative PCR (ChIP-qPCR) assays to examine the ASH1L occupancy, MLLAF9 occupancy, and histone $\mathrm{H} 3 \mathrm{~K} 36 \mathrm{me} 2$ modification at the gene promoters, transcriptional starting sites (TSS), transcriptional ending sites (TES) of Hoxa9 and Hoxa10, two MLL-AF9 target genes that were shown to be activated in the wild-type transformed cells and have reduced expression in the Ash1L-deleted cells (Figures 5B, E). The results showed that both ASH1L occupancy and histone H3K36me2 were enriched at the Hoxa9 and Hoxa10 promoters compared to that on the TES and the long terminal repeat (LTR) of intracisternal A-particle (IAP) (Figures 6A-E). Furthermore, compared to wild-type MLL-AF9-transformed cells, both ASH1L occupancy and histone H3K36me2 modification were reduced at the gene promoters in the $A \operatorname{sh} 1 L$-deleted cells (Figures 6A-E), suggesting that ASH1L bound to the Hoxa9 and Hoxa10 gene promoters directly and mediated local histone H3K36me2 modification. However, the MLL-AF9 occupancy at both gene promoters did not show significant difference between wild-type and Ash1L-deleted MLL-AF9-transformed cells (Figures 6F, G), suggesting the ASH1L-mediated histone H3K36me2 did not affect the binding of MLL-AF9 fusion protein to the gene promoters.

\section{DISCUSSION}

Chromosomal 11q23 translocations generate various MLL fusion proteins that contain the $N$-terminal portion of MLL1 and different fusion partners including AF9 (30, 31). Previous studies have demonstrated that the N-terminal MLL1 is critical for the recruitment of MLL fusion proteins to chromatin through its $\mathrm{CxxC}$-zinc finger (CxxC-zf) domain and its interacting proteins MENIN and LEDGF, while the $C$-terminal fusion partners interact with multiple trans-activators to induce 
A

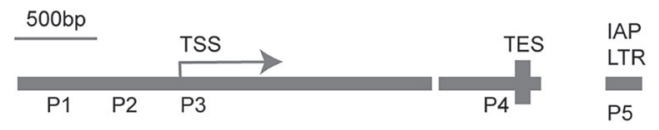

B

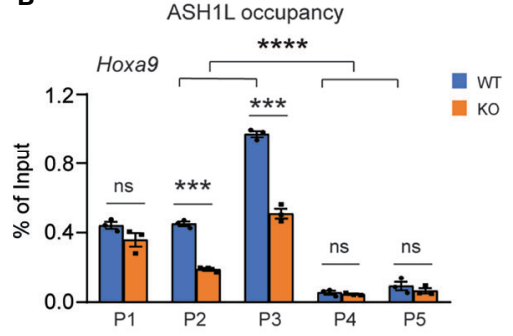

D

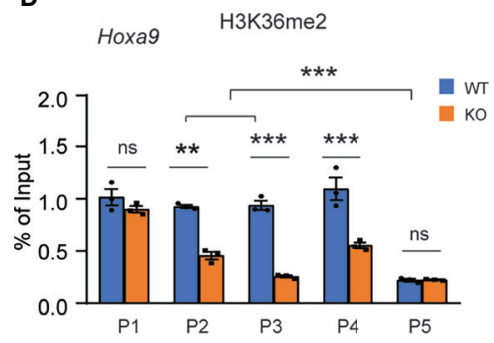

$\mathbf{F}$

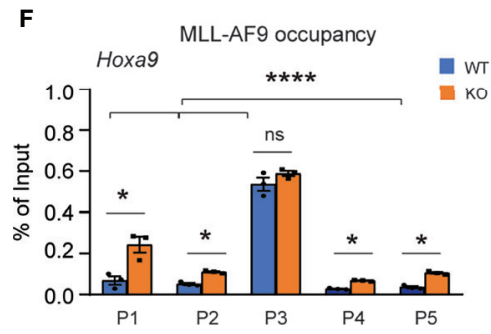

C

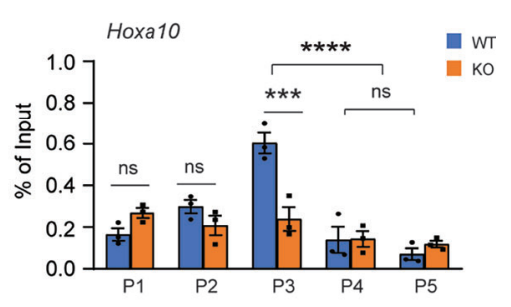

E

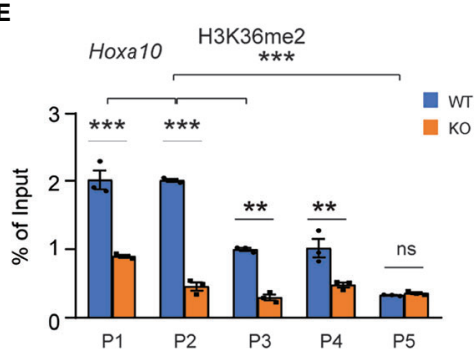

G

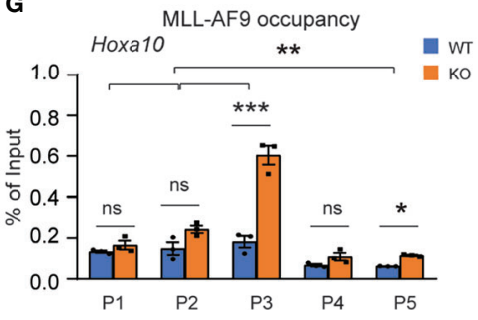

FIGURE 6 | ASH1L binds and mediates histone H3K36me2 modification at Hoxa9 and Hoxa10 gene promoters. (A) Plot showing the locations of ChIP-qPCR amplicons at the Hoxa9 and Hoxa10 gene loci and LTR of intracisternal A-particle (IAP). (B, C) ChIP-qPCR analysis showing the ASH1L occupancy at Hoxa9 and Hoxa10 gene loci in the wild-type and Ash1L-KO MLL-AF9-transformed cells. (D, E) ChIP-qPCR analysis showing the histone H3K36me2 at Hoxa9 and Hoxa10 gene loci in the wild-type and Ash1L-KO MLL-AF9-transformed cells. (F, G) ChIP-qPCR analysis showing the MLL-AF9 occupancy at Hoxa9 and Hoxa10 gene loci in the wild-type and Ash1L-KO MLL-AF9-transformed cells. Note: for panels (B-E), the error bars represent mean \pm SEM, $\mathrm{n}=3$ biological replicates. ${ }^{*} P<0.05$, ${ }^{\star \star} P<0.01,{ }^{* \star *} P<0.001 ;{ }^{* \star \star *} P<0.0001 ;$ ns, not significant.

transcriptional activation (16). Since the MLL fusion proteins lose the MLL1 C-terminal SET domain and its-associated histone H3K4 methyltransferase activity, it is unclear whether other histone KMTase-mediated histone modifications are required for the MLL fusion proteins to activate leukemogenic gene expression and induce leukemia development.

ASH1L is another member of TrxG proteins that facilitate transcriptional activation (8). Biochemically, ASH1L is a histone KMTase mediating histone H3K36me2 modification (20). Recent studies reported that ASH1L and MLL1 co-occupied the same gene promoters to activate gene expression, suggesting ASH1L and MLL function synergistically in activating gene expression in normal development and leukemogenesis (19, 21-23). However, the functional roles of ASH1L and its-mediated histone H3K36me2 in the MLLr-associated leukemogenesis have not been addressed using Ash1L gene knockout animal models.

In this study, we used an $A \operatorname{sh} 1 L$ conditional knockout mouse model to show that ASH1L and its histone methyltransferase activity are required for promoting the MLL-AF9-induced leukemogenesis. First, genetic deletion of ASH1L in normal HPCs largely impairs the MLL-AF9-induced colony formation in serial methylcellulose replating assays (Figure 1), suggesting ASH1L promotes the initiation of MLL-AF9-induced leukemic transformation. Second, loss of ASH1L in the MLL-AF9transformed cells largely impaired the colony formation in vitro and delayed the leukemia development in the recipient mice transplanted with leukemic cells (Figures 2 and 3), suggesting ASH1L facilitates the maintenance of MLL-AF9-transformed cells 
in vitro and leukemia progression in vivo. Importantly, the impaired ASH1L's function in the Ash $1 L-\mathrm{KO}$ cells could be rescued by the wild-type but not the catalytic-dead mutant ASH1L (Figure 4), suggesting that the histone methyltransferase activity is required for its function in promoting MLL-AF9-induced leukemogenic transformation, which is consistent with a recent study showing that the SET domain is required for the MLL-AF9-induced leukemic transformation (32).

At the cellular level, we observed that the loss of ASH1L in MLLAF9-transformed cells induced cell death and myeloid differentiation, which could be rescued by the wild-type but not the catalytic-dead mutant ASH1L (Figures 2, 4), suggesting that ASH1L promotes MLL-AF9-induced leukemic transformation though inhibiting cell apoptosis and blocking cell differentiation. The results are consistent with the molecular findings that ASH1L is required for the full activation of MLL-AF9 target genes including Hoxa gene cluster and MeisI (Figure 5), which are known to play important roles in leukemogenesis through inhibiting cell death and blocking normal cell differentiation (33-35). Finally, the ChIP assays showed that both ASH1L occupancy and histone H3K36me2 modification were enriched at the promoters of MLL-AF9 target genes Hoxa9 and Hoxa10 in the wild-type transformed cells (Figure 6), indicating the ASH1L regulates the MLL-AF9 target genes through directly chromatin binding and its-mediated histone H3K36me2 modification.

Previous studies have shown that the PWWP domain of LEDGF is required for the recruitment of MLL fusion proteins through its binding to histone $\mathrm{H} 3 \mathrm{~K} 36 \mathrm{me} 2(13,15,16)$. However, our ChIP analysis did not reveal reduction of MLL-AF9 occupancy at the Hoxa9 and Hoxa10 promoters in the Ash1LKO cells (Figures 6F, G), suggesting the MLL-AF9 fusion protein could bind to its target regions though other recruiting mechanisms, such as the $\mathrm{CxxC}$-zf domain-mediated binding to unmethylated CpG-rich promoters (36), and the reduced $\mathrm{H} 3 \mathrm{~K} 36 \mathrm{me} 2$ at gene promoters in the Ash $1 L$-KO cells impaired the Hoxa gene expression through mechanisms other than the recruitment of MLL-AF9 fusion protein.

Our current study has some limitations: (i) since this study includes a single type of MLLr, MLL-AF9 fusion protein, to induce leukemia development in mice, it is unclear whether ASH1L has the similar function in promoting other MLLr-induced leukemogenesis; (ii) although Ash1L deletion induces cell death, some MLL-AF9transformed cells survive in vitro and in vivo, suggesting the MLLAF9-transformed cells have heterogenous responses to the Ash $1 L$ depletion. However, the underlying mechanisms are not addressed by our current study. These fundamental questions merit further investigation for a better understating of the function of ASH1L in broad MLLr-associated leukemogenesis.

\section{REFERENCES}

1. Berger R, Bernheim A, Sigaux F, Daniel MT, Valensi F, Flandrin G. Acute Monocytic Leukemia Chromosome Studies. Leuk Res (1982) 6(1):17-26. doi: 10.1016/0145-2126(82)90039-X

2. Nakamura T, Alder H, Gu Y, Prasad R, Canaani O, Kamada N, et al. Genes on Chromosomes 4, 9, and 19 Involved in 11q23 Abnormalities in Acute
In summary, our study reveals that the histone H3K36me2specific methyltransferase ASH1L and its enzymatic activity play an important role in promoting the MLL-AF9-induced leukemogenesis, which provides an important molecular basis for targeting ASH1L and its enzymatic activity to treat MLLAF9-induced leukemias.

\section{DATA AVAILABILITY STATEMENT}

The datasets presented in this study can be found in online repositories. The names of the repository/repositories and accession number(s) can be found below: NCBI GEO; GSE183413.

\section{ETHICS STATEMENT}

The animal study was reviewed and approved by Michigan State University Institutional Animal Care \& Use Committee.

\section{AUTHOR CONTRIBUTIONS}

$\mathrm{JH}$ conceived the project. MA, YG, YW, and JH performed the experiments. $\mathrm{JH}$ and $\mathrm{GM}$ performed the sequencing data analysis. MA and $\mathrm{JH}$ interpreted the data and wrote the manuscript. All authors contributed to the article and approved the submitted version.

\section{FUNDING}

This work was supported by the National Institute Health NIH grant R01GM127431.

\section{ACKNOWLEDGMENTS}

MSU genomics core facility processed the nextgeneration sequencing.

\section{SUPPLEMENTARY MATERIAL}

The Supplementary Material for this article can be found online at: https://www.frontiersin.org/articles/10.3389/fonc.2021. 754093/full\#supplementary-material

Leukemia Share Sequence Homology and/or Common Motifs. Proc Natl Acad Sci U S A (1993) 90(10):4631-5. doi: 10.1073/pnas.90.10.4631

3. De Braekeleer M, Morel F, Le Bris MJ, Herry A, Douet-Guilbert N. The MLL Gene and Translocations Involving Chromosomal Band 11q23 in Acute Leukemia. Anticancer Res (2005) 25(3B):1931-44.

4. Vermaelen K, Barbieri D, Michaux JL, Tricot G, Casteels-Van Daele M, Noens L, et al. Anomalies of the Long Arm of Chromosome 11 in Human 
Myelo- and Lymphoproliferative Disorders. I. Acute Nonlymphocytic Leukemia. Cancer Genet Cytogenet (1983) 10(1):105-16. doi: 10.1016/01654608(83)90111-5

5. Ayton PM, Cleary ML. Molecular Mechanisms of Leukemogenesis Mediated by MLL Fusion Proteins. Oncogene (2001) 20(40):5695-707. doi: 10.1038/ sj.onc. 1204639

6. Chessells JM, Harrison CJ, Kempski H, Webb DK, Wheatley K, Hann IM, et al. Clinical Features, Cytogenetics and Outcome in Acute Lymphoblastic and Myeloid Leukaemia of Infancy: Report From the MRC Childhood Leukaemia Working Party. Leukemia (2002) 16(5):776-84. doi: 10.1038/ sj.leu. 2402468

7. Hilden JM, Dinndorf PA, Meerbaum SO, Sather H, Villaluna D, Heerema NA, et al. Analysis of Prognostic Factors of Acute Lymphoblastic Leukemia in Infants: Report on CCG 1953 From the Children's Oncology Group. Blood (2006) 108(2):441-51. doi: 10.1182/blood-2005-07-3011

8. Kingston RE, Tamkun JW. Transcriptional Regulation by Trithorax-Group Proteins. Cold Spring Harb Perspect Biol (2014) 6(10):a019349. doi: 10.1101/ cshperspect.a019349

9. Schuettengruber B, Chourrout D, Vervoort M, Leblanc B, Cavalli G. Genome Regulation by Polycomb and Trithorax Proteins. Cell (2007) 128(4):735-45. doi: 10.1016/j.cell.2007.02.009

10. Slany RK. The Molecular Biology of Mixed Lineage Leukemia. Haematologica (2009) 94(7):984-93. doi: 10.3324/haematol.2008.002436

11. Winters AC, Bernt KM. MLL-Rearranged Leukemias-An Update on Science and Clinical Approaches. Front Pediatr (2017) 5:4. doi: 10.3389/ fped.2017.00004

12. Chan AKN, Chen CW. Rewiring the Epigenetic Networks in MLL-Rearranged Leukemias: Epigenetic Dysregulation and Pharmacological Interventions. Front Cell Dev Biol (2019) 7:81. doi: 10.3389/fcell.2019.00081

13. Yokoyama A, Somervaille TC, Smith KS, Rozenblatt-Rosen O, Meyerson M, Cleary ML. The Menin Tumor Suppressor Protein is an Essential Oncogenic Cofactor for MLL-Associated Leukemogenesis. Cell (2005) 123(2):207-18. doi: 10.1016/j.cell.2005.09.025

14. Shun MC, Botbol Y, Li X, Di Nunzio F, Daigle JE, Yan N, et al. Identification and Characterization of PWWP Domain Residues Critical for LEDGF/p75 Chromatin Binding and Human Immunodeficiency Virus Type 1 Infectivity. J Virol (2008) 82(23):11555-67. doi: 10.1128/JVI.01561-08

15. Yokoyama A, Cleary ML. Menin Critically Links MLL Proteins With LEDGF on Cancer-Associated Target Genes. Cancer Cell (2008) 14(1):36-46. doi: 10.1016/j.ccr.2008.05.003

16. Yokoyama A. Transcriptional Activation by MLL Fusion Proteins in Leukemogenesis. Exp Hematol (2017) 46:21-30. doi: 10.1016/ j.exphem.2016.10.014

17. Okuda H, Kawaguchi M, Kanai A, Matsui H, Kawamura T, Inaba T, et al. MLL Fusion Proteins Link Transcriptional Coactivators to Previously Active CpG-Rich Promoters. Nucleic Acids Res (2014) 42(7):4241-56. doi: 10.1093/ nar/gkt1394

18. Jones M, Chase J, Brinkmeier M, Xu J, Weinberg DN, Schira J, et al. Ash1l Controls Quiescence and Self-Renewal Potential in Hematopoietic Stem Cells. J Clin Invest (2015) 125(5):2007-20. doi: 10.1172/JCI78124

19. Zhu L, Li Q, Wong SH, Huang M, Klein BJ, Shen J, et al. ASH1L Links Histone H3 Lysine 36 Dimethylation to MLL Leukemia. Cancer Discov (2016) 6 (7):770-83. doi: 10.1158/2159-8290.CD-16-0058

20. Yuan W, Xu M, Huang C, Liu N, Chen S, Zhu B. H3K36 Methylation Antagonizes PRC2-Mediated H3K27 Methylation. J Biol Chem (2011) 286 (10):7983-9. doi: 10.1074/jbc.M110.194027

21. Miyazaki H, Higashimoto K, Yada Y, Endo TA, Sharif J, Komori T, et al. Ash1l Methylates Lys36 of Histone H3 Independently of Transcriptional Elongation to Counteract Polycomb Silencing. PLoS Genet (2013) 9(11):e1003897. doi: 10.1371/journal.pgen.1003897

22. Gregory GD, Vakoc CR, Rozovskaia T, Zheng X, Patel S, Nakamura T, et al. Mammalian ASH1L Is a Histone Methyltransferase That Occupies the Transcribed Region of Active Genes. Mol Cell Biol (2007) 27(24):8466-79. doi: 10.1128/MCB.00993-07

23. Tanaka Y, Kawahashi K, Katagiri Z, Nakayama Y, Mahajan M, Kioussis D. Dual Function of Histone H3 Lysine 36 Methyltransferase ASH1 in
Regulation of Hox Gene Expression. PLoS One (2011) 6(11):e28171. doi: 10.1371/journal.pone.0028171

24. Gao Y, Duque-Wilckens N, Aljazi MB, Wu Y, Moeser AJ, Mias GI, et al. Loss of Histone Methyltransferase ASH1L in the Developing Mouse Brain Causes Autistic-Like Behaviors. Commun Biol (2021) 4(1):756. doi: 10.1038/s42003021-02282-z

25. Aljazi MB, Gao Y, Wu Y, Mias GI, He J. Cell Signaling Coordinates Global PRC2 Recruitment and Developmental Gene Expression in Murine Embryonic Stem Cells. iScience (2020) 23(11):101646. doi: 10.1016/ j.isci.2020.101646

26. He J, Shen L, Wan M, Taranova O, Wu H, Zhang Y. Kdm2b Maintains Murine Embryonic Stem Cell Status by Recruiting PRC1 Complex to CpG Islands of Developmental Genes. Nat Cell Biol (2013) 15(4):373-84. doi: $10.1038 / \mathrm{ncb} 2702$

27. Kim D, Pertea G, Trapnell C, Pimentel H, Kelley R, Salzberg SL. TopHat2: Accurate Alignment of Transcriptomes in the Presence of Insertions, Deletions and Gene Fusions. Genome Biol (2013) 14(4):R36. doi: 10.1186/ gb-2013-14-4-r36

28. Trapnell C, Hendrickson DG, Sauvageau M, Goff L, Rinn JL, Pachter L. Differential Analysis of Gene Regulation at Transcript Resolution With RNASeq. Nat Biotechnol (2013) 31(1):46-53. doi: 10.1038/nbt.2450

29. Ramirez F, Dündar F, Diehl S, Grüning BA, Manke T. deepTools: A Flexible Platform for Exploring Deep-Sequencing Data. Nucleic Acids Res (2014) 42 (Web Server issue):W187-91. doi: 10.1093/nar/gku365

30. Krivtsov AV, Armstrong SA. MLL Translocations, Histone Modifications and Leukaemia Stem-Cell Development. Nat Rev Cancer (2007) 7(11):823-33. doi: $10.1038 / \mathrm{nrc} 2253$

31. Dobson CL, Warren AJ, Pannell R, Forster A, Lavenir I, Corral J. The mll-AF9 Gene Fusion in Mice Controls Myeloproliferation and Specifies Acute Myeloid Leukaemogenesis. EMBO J (1999) 18(13):3564-74. doi: 10.1093/ emboj/18.13.3564

32. Rogawski DS, Deng J, Li H, Miao H, Borkin D, Purohit T, et al. Discovery of First-in-Class Inhibitors of ASH1L Histone Methyltransferase With AntiLeukemic Activity. Nat Commun (2021) 12(1):2792. doi: 10.1038/s41467-02123152-6

33. Sitwala KV, Dandekar MN, Hess JL. HOX Proteins and Leukemia. Int J Clin Exp Pathol (2008) 1(6):461-74.

34. Domsch K, Papagiannouli F, Lohmann I. The HOX-Apoptosis Regulatory Interplay in Development and Disease. Curr Top Dev Biol (2015) 114:121-58. doi: 10.1016/bs.ctdb.2015.07.014

35. Magli MC, Largman C, Lawrence HJ. Effects of HOX Homeobox Genes in Blood Cell Differentiation. J Cell Physiol (1997) 173(2):168-77. doi: 10.1002/ (SICI) 1097-4652(199711) 173:2<168::AID-JCP16>3.0.CO;2-C

36. Ayton PM, Chen EH, Cleary ML. Binding to Nonmethylated CpG DNA is Essential for Target Recognition, Transactivation, and Myeloid Transformation by an MLL Oncoprotein. Mol Cell Biol (2004) 24 (23):10470-8. doi: 10.1128/MCB.24.23.10470-10478.2004

Conflict of Interest: GM has consulted for Colgate-Palmolive North America.

The remaining authors declare that the research was conducted in the absence of any commercial or financial relationships that could be construed as a potential conflict of interest.

Publisher's Note: All claims expressed in this article are solely those of the authors and do not necessarily represent those of their affiliated organizations, or those of the publisher, the editors and the reviewers. Any product that may be evaluated in this article, or claim that may be made by its manufacturer, is not guaranteed or endorsed by the publisher.

Copyright (๑) 2021 Aljazi, Gao, Wu, Mias and He. This is an open-access article distributed under the terms of the Creative Commons Attribution License (CC BY). The use, distribution or reproduction in other forums is permitted, provided the original author(s) and the copyright owner(s) are credited and that the original publication in this journal is cited, in accordance with accepted academic practice. No use, distribution or reproduction is permitted which does not comply with these terms. 